71 ANALYSING THE IMPACT OF VIRTUAL TEACHING SESSIONS ON LEARNING EXPERIENCES OF PHYSICIANS DURING THE COVID-19 ERA AT CHI

Liqa ur Rehman*. Children's Health Ireland

10.1136/archdischild-2021-europaediatrics.71

To determine how virtual teaching sessions have impacted learning experiences of physicians during the COVID-19 era at $\mathrm{CHI}$.

We created an anonymous survey on Google forms with a fixed time frame to complete. Our target population included all physicians working at $\mathrm{CHI}$.

Our questions focused on

1) Individual educational resources available across $\mathrm{CHI}$

2) External webinars

3) Accessibility around the applications used to broadcast the virtual sessions Data was collected prospectively and was analysed availing Micro-soft Excel -We received a total of 71 respondents with a mix of sites and grades.

-Responses to individual sessions including journal club and grand rounds were generally positive, with some negative feedback around accessibility, interaction and quality of IT Over half of all participants were relying on their personal phone (63.4\%) and mobile data (69\%) rather than hospital IT equipment

- Most popular session to continue online was cross-city grand rounds (63.4\%).

Only $10 \%$ of respondents did not want to continue any sessions virtually post pandemic According to the results from the questionnaire above, virtual learning experiences for NCHDs and consultants were positive, with most very satisfied with the ease of access. However, further improvements in the IT department at CHI and addition of radiology meetings could be added to further enhance learning experiences.

\section{PAEDIATRIC MULTISYSTEM INFLAMMATORY SYNDROME, A NEW ENTITY: A 3-CASE SERIES}

Joana Filipe Ribeiro*, Alexandra M Rodrigues, Ana Catarina Francisco, Pedro Carvalho, Pedro Fernandes, Pedro Guerra. Department of Pediatrics, Hospital Sousa Martins, ULS Guarda

\subsection{6/archdischild-2021-europaediatrics.72}

Introduction/Description Covid-19 is usually mildly symptomatic in children. However, in April 2020 the first reports of an inflammatory generalized syndrome associated with

SARS-CoV-2 emerged. We report 3 cases of this new entity in a secondary hospital.

A 9-year-old female, with a low-risk contact with SARSCOV 2 infected person, was admitted to the Emergency Department (ED) due to persistent fever with lasting for 6 days, fleeting rash at the trunk, oral mucositis, cheilitis and painful cervical lymphadenopathies. Laboratory studies showed leucocytosis, elevation of erythrocyte sedimentation rate (ESR), C-Reactive Protein (CRP), D-Dimers and fibrinogen. Echocardiography depicted hyperechogenicity in the coronary arteries. Treatment with a single dose of intravenous immunoglobulin (IVIg) and aspirin (ASA) was performed, but clinical worsening motivated the introduction of oral corticotherapy. She was discharged after 8 days with clinical and analytical improvement.

An 8-year-old female, infected with SARS-COV-2 twenty days before the admission, presented to the ED complaining of persistent fever with a 2-days evolution, associated with abdominal pain and maculopapular rash in the trunk and face. Laboratory data showed lymphopenia, thrombocytopenia and elevation of inflammatory markers (CRP, ESR and ferritin), DDimers, fibrinogen and cardiac enzymes. Echocardiography revealed hyperechogenicity of the coronary arteries. She began treatment with IV methylprednisolone pulses for 3 days, ASA and oral corticoid. A clinical and analytical improvement was observed and she was discharged after 8 days of hospitalization.

A 12-year-old female, infected with SARS-COV 2 one month before, was admitted to the ED due to persistent fever with a 2-days evolution.

Analytical study revealed lymphopenia, thrombocytopenia, elevation of CRP and D-Dimers. The child was hospitalized for clinical surveillance. After 5 days of hospitalization, she referred more tiredness associated with persistent fever and bilateral non-exudative conjunctivitis. Laboratory data showed elevation of inflammatory markers and D-Dimers, worsening of thrombocytopenia, hypoalbuminemia and elevation of troponin.

Echocardiography evaluation was normal. IVIg and oral ASA was initiated with analytical and clinical improvement. In this case, genomic sequencing was possible and the variant SARS-COV 2 B.1.221 was identified.

Conclusion Paediatric Multisystem Inflammatory Syndrome temporally associated with

Covid-19 (PIMS-TS) is a new entity not always a straightforward diagnosis.

Considering the virus dissemination, it is hard to keep track of the potential high-risk contacts. Therefore, we should be aware of PIMS-TS to archive an early diagnosis and potentially avoid severe complications. There is still insufficient data to conclude if certain variants are more susceptible to cause this syndrome.

\section{ABDOMINAL PAIN IN POST-COVID 19 PEDIATRIC PATIENTS}

Nadezhda Rimpova*, Mariana Ardalieva, Galina Angelova. University Hospital 'Sofiamed'

\subsection{6/archdischild-2021-europaediatrics.73}

The coronavirus pandemic first broke in December 2019. Unlike adults, children have been reported to present with milder clinical manifestations of the virus, sometimes even acting as asymptomatic carriers of infection.

However, as early as mid-2020, pediatric patients who developed multisystem inflammatory syndrome (MIS-C), along with fever, cardiorespiratory symptoms and neurocognitive problems, were found to exhibit marked gastrointestinal (GI) manifestations, sometimes confounding diagnosis by mimicking GI infections, inflammatory bowel disease (IBD) or surgical contitions, such as acute appendicitis.

We present series of cases of the school age children, who presented with severe abdominal pain during Bulgaria's first peak of COVID-19 pandemic. 
Characteristic symptoms of the disease - disosmia, nausea, vomiting, loss of appetite were reported to occur 2 weeks prior to hospitalization and were only mild or barely causing any concern to the parent. Children were admitted with symptoms of mild to moderate dehydration, metabolic acidosis and often constipation. The classical laboratory findings of elevated inflammatory response, leucopenia and lymphopenia were absent. Abdominal ultrasound was negative. Repeated surgical exams revealed no signs of acute abdomen. Qualitative antibody testing showed either presence of both $\operatorname{IgM}$ and IgG antibodies against SARS CoV-2 or only IgG antibodies. Some children had pathologic urinary findings, such as hematuria. Signs of autonomic dysfunction were observed since all of the patients had bradycardia and variations of blood pressure. No respiratory symptoms were registered and no children Had no history of pre-existing conditions.

Symptomatic medication was effective only in some patients. Empiric AB-treatment proved to be much more successful, though no causative agent of intestinal infection was isolated.

Manifestations of post-infectious MIS-C, associated with COVID-19 often include gastro-intestinal symptoms. For definitive diagnosis of the condition, tests for COVID-19 should be administered, since not all laboratory findings might be consistent with case definitions.

\section{CHILDREN'S PERSPECTIVE: HOW TO STAY 'NORMAL' IN 'ABNORMAL' WORLD}

Ana Goleš*, Tamara Milovanović, Danijela Vuković Lerga, Iva Kirša. Clinical Hospital Center Rijeka

\subsection{6/archdischild-2021-europaediatrics.74}

The coronavirus pandemic has conquered the world in 2020 and had an outstanding impact on our everyday lives. Face mask mandates, constant use of disinfectants, social distancing, limited movement, working from home and online teaching are only some of the changes that we had to incorporate in our lives. Regardless of age, gender, occupation or socio-economic status, there is no single individual who was not challenged to adapt to the new circumstances.

Children and adolescents are much more vulnerable to the effects of any event that disrupts or limits how they function. This can cause long-term damage to the health and quality of their upbringing, as well as their proper socio-emotional and physical development.

In order to gain insight into the effects of the pandemic on the emotional status of children and adolescents, an online questionnaire was distributed to participants aged 10 to 18 in two periods - spring of 2020 and spring of 2021 .

We believe that the acquired data can contribute to a better understanding of the effects of the pandemic on the lives of children and adolescents by analyzing any disruptions in mood, consequences on interpersonal relations, capacity to adapt and assessing quality of life.

Better understanding leads to a better ability to define the critical areas in which to provide professional help and support. It can also provide key guidelines for preventing developmental disruptions in vulnerable groups such as children and adolescents.

\section{HOW ACTIVE IS ACTIVE? PHYSICAL ACTIVITY LEVELS IN CHILDREN LIVING WITH OBESITY}

A McClean*, J Gordon, J McGoldrick, A Long, J Gawley, A Harbinson, N Khan, R Graham, B O'Connor. Nutrition Group, Centre for Public Health, Queen's University Belfast

\subsection{6/archdischild-2021-europaediatrics.75}

Background and Aims Physical activity is essential for healthy growth and development. Being active is an important tool for the prevention and treatment of childhood obesity. Physical activity can benefit children with obesity by helping them build a healthier body composition including stronger bones and muscles and increasing their energy expenditure. Other benefits for physical activity in children with obesity include improving mental wellbeing, sleep, gross motor skills and energy levels.

The UK Chief Medical Officers (CMO) recommend that children aged 5-18 years should engage in moderate-to-vigorous intensity physical activity for at least 60 minutes per day. Moderate-to-vigorous intensity activities are defined as requiring effort and noticeably raising the heart and breathing rates. Examples include cycling, jumping and active play.

This aim of this project was to assess physical activity levels in a population of children aged 5-16 years living with obesity and survey parental knowledge of the $\mathrm{CMO}$ guidelines.

Methods Families with a child aged 5-16 years living with obesity (defined by a BMI greater than the 98th centile on the RCPCH Growth Charts) were interviewed by a paediatric physiotherapist. Parents/carers were asked 3 questions.

'Do you consider your child to be physically active?' 'How many minutes of physical activity do you think are recommended by the UK CMOs for your child based on his/her age?' The third question was 'Can you describe your child's daily routine including any physical activities?'

Families described their child's daily routine including play, sport, and active travel. Answers were recorded by the physiotherapist, anonymously complied, and analysed by the authors. Results Families of 47 children living with obesity participated. $53 \%$ of parents/carers answered 'Yes' that they considered their child to be physically active. 47\% answered 'No.' Only $19 \%$ of parents answered correctly when they were asked how many minutes of daily activity was recommended by the UK CMO. Upon analysis of the family accounts of their child's daily activities only 5 of the 47 children (12\%) were achieving their daily physical activity targets.

Conclusions Encouraging children who are living with obesity to engage in physical activity is a fundamental treatment goal. This project highlights that a low percentage of children (12\%) living with obesity are achieving the recommended daily activity levels. Many parents of children with obesity are not aware of the correct CMO recommendations for physical activity and overestimate how active their child is. As healthcare professionals we should promote and educate parents about the $\mathrm{CMO}$ recommendations.

\section{A PRIMARY CARE APPROACH TO PAEDIATRIC MENTAL HEALTH DISORDERS IN IRELAND}

Edmond Power*, Farhana Sharif. School of Medicine, University College Dublin

10.1136/archdischild-2021-europaediatrics.76 